\title{
L’Exécutif écossais en guerre
}

Stratégies de lutte contre le sectarisme religieux

\section{Nathalie Duclos}

\section{(2) OpenEdition}

Journals

Édition électronique

URL : http://journals.openedition.org/etudesecossaises/209

DOI : 10.4000/etudesecossaises.209

ISSN : 1969-6337

Éditeur

UGA Éditions/Université Grenoble Alpes

Édition imprimée

Date de publication : 31 mars 2005

Pagination : 257-268

ISBN : 2-84310-061-5

ISSN : 1240-1439

Référence électronique

Nathalie Duclos, "L'Exécutif écossais en guerre », Études écossaises [En ligne], 10 | 2005, mis en ligne le 31 mars 2005, consulté le 08 septembre 2020. URL : http://journals.openedition.org/ etudesecossaises/209; DOI : https://doi.org/10.4000/etudesecossaises.209 


\section{L'exécutif écossais en guerre Stratégies de lutte contre le sectarisme religieux}

NATHALIE Duclos Université Toulouse Le Mirail

1. Cross-Party Working Group on Religious Hatred, Tackling Religious Hatred, Édimbourg, The Stationery Office, 2002 2. "It is time for the decent majority of Scots to stand up and be counted and to say that religious hatred should be put in the dustbin of history. It is time for Scotland's secret shame to be put in the past.»
Dans les jours qui suivirent les attentats du 11 septembre 2001, plusieurs mosquées écossaises subirent des dégradations. Ces agressions antimusulmanes relancèrent la réflexion sur la haine religieuse dans cette région du Royaume-Uni et provoquèrent la mise en place, en novembre 2001, d'un groupe de travail transpartisan au sein du Parlement écossais. Le CrossParty Working Group on Religious Hatred fut chargé de déterminer si l'intolérance religieuse pouvait être combattue par des moyens législatifs et de considérer des propositions de réformes à cet effet. Le rapport final du groupe de travail, intitulé Tackling Religious Hatred, parut un an plus tard, le 5 décembre $2002^{1}$. Le jour même, le Premier ministre Jack McConnell déclara que le sectarisme religieux était la «honte secrète de l'Écosse ${ }^{2} \gg$ et que désormais, la lutte contre cette forme d'intolérance constituerait l'une des priorités nationales. Cette prise de position, que beaucoup jugèrent excessive ou même injustifiée, soulève plusieurs questions. D'une part, à quoi le chef de l'exécutif écossais faisait-il référence lorsqu'il parlait de sectarisme religieux? Peu de ses compatriotes sont réellement convaincus que la société écossaise dans son ensemble soit sectaire et la plupart pensent plutôt que l'intolérance religieuse est l'apanage des habitants des quartiers pauvres de la région de Glasgow, voire des supporters de certaines équipes de football de tradition sectaire. Il s'agit donc, dans un premier temps, de cerner le phénomène du sectarisme religieux tel qu'il se manifeste en Écosse et d'expliquer pourquoi les déclarations de Jack McConnell ont fait débat dans le pays. D'autre part, quelles stratégies l'exécutif se proposait-il de mettre en œuvre pour lutter contre le sectarisme religieux? À ce jour, le gouvernement a déjà adopté certaines des douze suggestions énoncées dans le rapport de décembre 2002 sur la haine religieuse. Il a surtout fait voter un article de loi faisant de la haine religieuse une circonstance aggravante dans le jugement d'un délit ou d'un crime. 


\section{Définir le sectarisme écossais : remarques historiques sur le sectarisme politico-religieux}

Dès l'été 1999, le compositeur écossais James MacMillan avait fait scandale en prononçant à l'occasion du Festival international d'Édimbourg un discours intitulé «Scotland's Shame», termes que devaient reprendre Jack McConnell plusieurs années plus tard. Dans ce discours désormais célèbre, le compositeur, qui est de confession catholique, s'était élevé contre ce qu'il percevait comme les tendances manifestement anticatholiques de la société écossaise. De la même manière, le rapport du Cross-Party Working Group on Religious Hatred, pourtant fondé après les attentats du 11 septembre 2001, ne fait que marginalement référence à la question des agressions subies par la communauté musulmane, et se concentre plutôt sur le problème du sectarisme inter-chrétien, qui opposerait certains Écossais de confession protestante, principalement des membres de différentes Églises presbytériennes, notamment l'Église d'Écosse, à leurs compatriotes catholiques, pour la plupart descendants d'Irlandais arrivés en Écosse dans la seconde moitié du XIX ${ }^{e}$ et au début du XX ${ }^{e}$ siècle. Aujourd'hui, l'Écosse compte environ 800000 catholiques, parmi lesquels

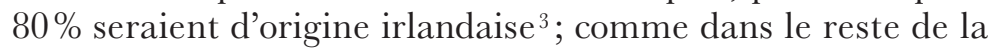
Grande-Bretagne, la communauté catholique constituerait donc près de $17 \%$ de la population écossaise totale. Toutefois, lorsqu'on parle d'intolérance religieuse inter-chrétienne dans

3. Cette dernière estimation provient de l'ouvrage suivant: I. R. Paterson, «The Pulpit and the Ballot Box: Catholic Assimilation and the Decline of Church Influence» (Devine, 2000, p. 228).

4. Les mariages mixtes représenteraient à peine $5 \%$ des mariages en Ulster, alors que selon les régions, ils représenteraient entre une bonne moitié et la grande majorité des mariages écossais (Mulholland, 2002, p. 223).

5. S. Bruce, «Hooliganism Is the Real Source of the Problem » Herald, 6 décembre 2002. le contexte britannique, c'est presque toujours en référence à l'Irlande du Nord et non à l'Écosse, et l'idée que certaines formes de sectarisme puissent survivre en Écosse fait débat. Steve Bruce, professeur de sociologie à l'université de Aberdeen et spécialiste de la question, souligne par exemple que la société écossaise, contrairement à la nord-irlandaise, ne se définit pas par ce clivage religieux; les mariages mixtes y sont notamment chose courante, alors qu'ils restent très minoritaires en Irlande du Nord ${ }^{4}$. Par ailleurs, si le sectarisme religieux a sans conteste représenté un vrai problème de société à la fin du XIX et au début du XX ${ }^{\mathrm{e}}$ siècle, au plus fort de l'immigration irlandaise, et surtout dans l'entre-deux-guerres, lorsque l'ordre d'Orange connut en Écosse ses heures de gloire, Bruce rappelle qu'il a nettement décliné dans le pays depuis la fin des années $1930^{5}$. Certains sont néanmoins d'avis que le sectarisme a refait surface en Écosse depuis les années 
1970 et la reprise des «troubles» en Irlande du Nord. À l'appui de cette théorie, on peut évoquer les deux attentats dont des pubs de Glasgow furent la cible en 1979, et qui furent commis par deux frères, Colin et William Campbell, membres de l'une des sections locales de l'Ulster Volunteer Force, célèbre organisation paramilitaire protestante. Notons toutefois que ces attentats n'ont heureusement fait aucune victime, et qu'ils sont restés des actes isolés, puisqu'ils demeurent à ce jour les seuls actes terroristes sectaires commis sur le territoire écossais. Quant aux agressions d'origine religieuse, jusqu'en juin dernier, il était très difficile d'en évaluer le nombre, car elles n'étaient recensées ni par les services de police, ni par les tribunaux, et seuls deux incidents récents étaient unanimement reconnus comme ayant une telle origine. Le plus célèbre d'entre eux reste le meurtre de Mark Scott à Glasgow, le 7 octobre 1995. Pour avoir porté le maillot du Celtic de Glasgow, club de football de tradition irlandaise et catholique, cet adolescent âgé de 16 ans à peine fut égorgé à son retour d'un stade de football par un jeune supporter du club rival de tradition protestante, les Glasgow Rangers. Ce meurtre a donné lieu à la fondation de Nil By Mouth, qui est aujourd'hui la principale association de lutte contre la haine religieuse en Écosse. Le second incident est très similaire au premier: le 31 mars 1999, Thomas McFadden, supporter du Celtic, lui aussi âgé de 16 ans, fut tué par deux supporters des Rangers à sa sortie d'un pub de Glasgow où il avait suivi un match de football opposant les deux équipes. En l'absence de statistiques officielles sur le nombre d'agressions religieuses commises en Écosse, ces deux meurtres sont les seuls dont personne ne conteste l'origine sectaire. Deux attentats et deux meurtres sectaires en vingt ans: peut-on vraiment parler de «sectarisme» dans le cas écossais, d'autant que certains de ces incidents sont liés, souligne Steve Bruce, puisque l'assassin de Mark Scott, nommé Jason Campbell, n'est autre que le fils de l'un des frères Campbell responsables des attentats de 1979? Bruce en conclut que l'intolérance religieuse reste en Écosse un phénomène très marginal ${ }^{6}$.

Pourtant, il semblerait que la population écossaise soit presque unanimement persuadée de la survivance du sectarisme dans le pays. Un sondage de l'institut ICM Research nous apprend que, en février 1998, 89\% des Écossais considéraient encore le sectarisme comme un véritable problème de 
société 7 . Aujourd'hui, beaucoup d'entre eux pensent toutefois qu'il ne survit guère que dans l'ouest du pays, notamment à Glasgow, et dans les villes industrielles des alentours, où la population catholique d'origine irlandaise constitue près du quart de la population totale. En outre, certains estiment que le sectarisme dans sa version écossaise se limite aux provocations et agressions entre supporters de clubs de football rivaux de tradition catholique d'un côté et protestante de l'autre, c'est-à-dire notamment les deux principaux clubs de Glasgow, les Rangers et le Celtic, mais aussi les deux clubs rivaux d'Édimbourg, les Hearts (ou Heart of Midlothian), club protestant, et les Hibs (ou Hibernian), club catholique. Il existe en Écosse un dernier club de tradition irlandaise et catholique: c'est l'actuel Dundee United, qui s'est appelé par le passé Dundee Harp, puis Dundee Hibernian. Tout match opposant un club de tradition catholique à un club de tradition protestante est donc potentiellement le lieu de manifestations d'ordre sectaire, mais jamais l'intolérance religieuse n'est aussi visible et aussi bien partagée que lors des matchs opposant les Rangers au Celtic, qui sont non seulement les deux meilleurs clubs écossais (et de loin), mais qui sont aussi les plus anciens clubs de Glasgow, ce qui leur vaut d'être qualifiés d'Old Firm. Devant le stade où se joue le match Rangers-Geltic, des vendeurs à la sauvette proposent des articles à la gloire et aux couleurs des deux équipes, mais aussi des articles plus ligieux, qui renvoient au conflit nord-irlandais, tels que des drapeaux ou des bannières, des écharpes, des T-shirts ou même des articles paramilitaires. Pendant le match, des milliers de supporters des deux équipes entonnent des chants provocateurs, dont l'hymne national irlandais d'un côté, l'hymne britannique ou Rule Britannia de l'autre, mais aussi certains chants engagés irlandais qui appellent ouvertement à la violence sectaire. Les chants des supporters du Celtic sont souvent des chansons folkloriques irlandaises, mais certains d'entre eux sont plus controversés, comme par exemple The Boys of the Old Brigade, qui relate l'histoire d'un père rejoignant les rangs de l'IRA. Du côté des supporters des Rangers, parmi les chants les plus ouvertement politiques, on peut notamment citer The Sash My Father Wore, qui est un des chants traditionnels des défilés orangistes et qui fait référence à la victoire de Guillaume d'Orange face aux

7. <http://www.icm research.com $>$. troupes du roi catholique Jacques VII d'Écosse et II d'Angleterre lors de la bataille de la Boyne, le 12 juillet 1690, ou le 
8. Deux versions de ce chant, l'une traditionnelle et l'autre sectaire, sont reproduites en annexe au présent article.

9. Il s'agit de la phrase "We're up to our knees in Fenian blood», dans laquelle «Fenian» est un terme hautement péjoratif signifiant catholique. En 1858, John O’Mahony, un révolutionnaire irlandais arrivé en Amérique cinq ans auparavant pour convertir à sa cause les immigrés irlandais, devint le chef d'une nouvelle organisation, la Fenian Brotherhood, quelques mois après la fondation à Dublin d'une société secrète, l'Irish Republican Brotherhood, par un autre révolutionnaire James Stephen. Le terme «Fenian» fut ensuite utilisé pour désigner l'ensemble

des conspirateurs révolutionnaires, puis l'ensemble des catholiques. La Fenian

Brotherhood tirait ellemême son nom d'une bande de guerriers menée par un héros gaélique légendaire nommé Finn Mac Cool (ou Fionn Mac Cumhaill, ou Find Mac Ctjmaill).

10. "What if, instead of «Fenian", the word 'nigger'had been used [...] would the politicians have stood through proceedings dominated by chanting of thousands who wanted to be up to their knees in nigger blood?"

<http://www.realmaroonfc.com/documents/ eat_sectarianism_l.htm

11. Le nationaliste écossais Jim Sillars avait noté à son grand regret que les Écossais ne se redécouvraient patriotes que pendant les matchs Écosse-Angleterre: chant Hullo, Hullo qui, dans sa version détournée ${ }^{8}$, inclut une phrase qui appelle ouvertement à la violence sectaire ${ }^{9}$.

Peut-on qualifier de sectaire l'attitude de ces supporters? Pour beaucoup d'entre eux, ces chants ne sont guère que des mots, et, à ce titre, ne représentent pas de véritables agressions sectaires. Cet argument, qui consiste à dire que le sectarisme n'est pas dangereux ni condamnable dès lors qu'il reste verbal, n'est bien sûr pas défendable. Jimmy Reid, ancien dirigeant du Communist Party of Great Britain et aujourd'hui partisan du Scottish Socialist Party, a par exemple dénoncé le fait que, jusqu'à très récemment, les institutions politiques étaient prêtes à considérer comme de véritables agressions les attaques verbales d'origine raciale, par exemple, mais non celles d'origine religieuse. Faisant allusion à la fameuse phrase «We're up to our knees in Fenian blood», il s'est notamment demandé si la classe politique n'aurait pas été plus prompte à condamner le chant Hullo, Hullo et les milliers d'Écossais qui l'entonnent s'il avait contenu le terme «Nègre» («Nigger») au lieu du terme «Fenian $»^{10}$. Dans un tel contexte, on comprend que l'un des principaux objectifs de l'association Nil By Mouth soit d'obtenir des Écossais la reconnaissance du fait que les mots peuvent à la fois être une forme de violence en soi et conduire à la violence physique. Par ailleurs, de nombreux Écossais sousestiment la gravité du phénomène sous prétexte que l'immense majorité des supporters appelant à la violence sectaire pendant les matchs mettent leur sectarisme entre parenthèses lorsqu'ils rentrent chez eux. Le patriotisme écossais a dans le passé été qualifié de «patriotisme de 90 minutes ${ }^{11}$ »; le sectarisme écossais serait-il lui aussi un «sectarisme de 90 minutes»? Selon les tenants de ce point de vue, l'attitude extrême de certains supporters relèverait davantage du hooliganisme que de l'intolérance religieuse à proprement parler. Dans un article précisément intitulé «Hooliganism is the Real Source of the Problem», publié le lendemain des déclarations de Jack McConnell sur le sectarisme, Steve Bruce a par exemple affirmé que les actes de violence qualifiés de sectaires étaient tout autant, voire davantage, motivés par la pauvreté, l'alcool, la drogue ou le manque d'éducation que par l'intolérance religieuse ${ }^{12}$. Cette dernière ne concernerait que les hooligans et la jeunesse défavorisée et désouvrée; elle ne serait donc pas un problème de société. Certains incidents récents semblent néanmoins contredire cette logique. On peut notamment citer 
«The great problem is that Scotland has too many 90-minute patriots whose nationalist outpourings are expressed only at major sporting events.»

12. S. Bruce, art. cit.: «What troubles me about this debate is an inability to distinguish two separate issues: casual violence and religious intolerance. The west coast of Scotland is blighted by hooliganism. We should be deeply troubled that so many young men drink too much, take drugs, carry sharp weapons, and like violence. Just because some of that violence occurs at Old Firm football matches and is glorified by the language and symbols of competing national and religious identities does not mean that sectarian animosity is a major social issue in Scotland.»

13. "The issue is too easily characterised as a problem in Scottish society that is associated only with football and with two football teams in particular. However, the issue runs much deeper than that and is much more insidious. In the football context, the problem is at least open and obvious, but a great deal of hidden sectarianism permeates a large part of Scottish society. » Les compte rendus des réunions de commissions parlementaires sont reproduits su le site internet du Parlement écossais à l'adresse suivante:

$<$ http://www.scottish.pa rliament.uk>. 14. Ce ministère est dirigé par le Lord Advocate, qui est secondé par le Solicitor General for Scotland. le scandale qui a frappé les Rangers en mai 1999, quand la presse a révélé que Donald Findlay, le vice-président du club, avait chanté The Sash My Father Wore lors d'une réception donnée pour célébrer la victoire des Rangers contre le Celtic en finale de la Coupe d'Écosse. Cet incident, qui s'est produit le même soir que le meurtre de Thomas McFadden par deux supporters des Rangers, a convaincu beaucoup d'Écossais que l'intolérance religieuse existait à tous les niveaux de la société, et non pas seulement au sein de la classe ouvrière et chez les personnes les moins éduquées. Notons qu'en plus d'être vice-président des Rangers, Donald Findlay était à la fois avocat de la Couronne (Queen's Counselor) et recteur de la vénérable université de St Andrews, poste dont il dut d'ailleurs démissionner à la suite de cet incident. Tous les Écossais ne partagent donc pas le point de vue de Steve Bruce sur le sectarisme religieux. Scott Barrie, député travailliste au Parlement d'Édimbourg, a par exemple affirmé, lors d'une réunion de la commission à la justice à laquelle il appartenait, que le phénomène du sectarisme était plus généralisé qu'on l'avait souvent affirmé par le passé ${ }^{13}$. Labsence de consensus concernant l'étendue du sectarisme dans le pays ou même la signification des comportements sectaires a longtemps justifié le mutisme des institutions politiques sur la question. Ce fut donc un véritable coup de théâtre lorsque, le 5 décembre 2002, le Premier ministre et son ministre de la Justice, Jim Wallace, annoncèrent la mise en œuvre de plusieurs mesures, d'un "plan d'action» selon les termes de Jim Wallace, destiné à la fois à mieux cerner le phénomène du sectarisme en Écosse, et à mieux lutter contre ses manifestations.

\section{La lutte contre le sectarisme: les mesures préconisées par l'exécutif écossais}

Ce plan d'action s'inspirait largement des douze recommandations qui concluaient le rapport du groupe de travail sur la haine religieuse. Leurs première et troisième recommandations concernaient la mise en place d'un système de recensement de toutes les agressions à caractère religieux, par la police d'une part, et par la justice d'autre part. Le Crown Office and Procurator Fiscal Service, qui est une branche de l'exécutif écossais ${ }^{14}$, devait notamment recenser à la fois toutes 
les agressions à caractère religieux portées devant les tribunaux, et l'issue des procès: il s'agissait de déterminer si le mobile religieux était systématiquement pris en compte dans le jugement d'une affaire. Dans sa deuxième recommandation, le groupe de travail proposait que les procureurs, ou Procurators Fiscal, soient systématiquement informés du caractère sectaire d'une agression, comme c'est le cas pour les crimes à caractère racial notamment; il condamnait ainsi une pratique, autrefois courante, selon laquelle les procureurs pouvaient abandonner le chef d'accusation «agression à caractère religieux» si l'accusé acceptait de plaider coupable pour un délit de moindre importance. Dans sa quatrième recommandation, le groupe de travail conseillait à l'exécutif de lancer un programme de recherches sur la haine religieuse, afin de mieux cerner le phénomène du sectarisme et d'en proposer une définition scientifique. Les recommandations cinq à huit concernaient la Scottish Football Association et les clubs de football. Le rapport suggérait que la SFA n'accorde de licence qu'aux clubs ayant développé une politique de lutte contre le sectarisme, et qu'elle pénalise tous ceux qui ne se tenaient pas à cette politique. Les clubs eux-mêmes devaient se charger de pénaliser leurs supporters à l'attitude sectaire, en les privant d'abonnement pour toute une saison par exemple, ou de façon définitive pour ceux coupables d'une agression particulièrement grave. La police devait également informer les clubs chaque fois qu'elle arrêtait un de leurs supporters. Enfin, les matchs Rangers-Celtic devaient continuer à être programmés plus tôt que les autres, la police ayant constaté qu'une telle mesure faisait diminuer le nombre d'agressions commises après les matchs. La neuvième recommandation visait les collectivités locales, notamment le conseil local de Glasgow. Le groupe de travail jugeait souhaitable que les conseils locaux interdisent la vente devant les stades d'articles paramilitaires et d'articles incitant à la violence sectaire. Aujourd'hui, le comité aux licences du Glasgow City Council envisage donc de délivrer une licence à tous les vendeurs de rue de façon à pouvoir exercer un contrôle sur la marchandise vendue devant les stades. Dans ses trois recommandations finales, le groupe de travail se prononçait en faveur d'une plus grande coopération entre la police, l'exécutif, les collectivités locales, les organisations telles que Nil By Mouth et les clubs de football, qui devaient financer des projets communs de lutte contre la haine 
15. Notons qu'une telle campagne pourrait s'inspirer de la courte mais populaire campagne d'affichage organisée par Nil By Mouth dans la ville de Glasgow à l'été 2000. Sur l'une de leurs affiches, on pouvait par exemple voir un visage couvert de cicatrices, accompagné du slogan: Sectarian jokes can have you in stitches.

16. Amendement 148 au Criminal Justice (Scotland) Bill. Justice 2 Committee, Official Report, Col. 2470, 49th meeting (2002), session 1 . religieuse. Il conseillait également à l'exécutif de lancer sa propre campagne de lutte contre le sectarisme ${ }^{15}$.

Dès décembre 2002, l'exécutif s'est déclaré favorable à l'ensemble des recommandations du groupe de travail. Certaines d'entre elles ont déjà été adoptées. L'association Nil By Mouth, en partenariat avec le conseil municipal de Glasgow, le Celtic, les Rangers, l'Église catholique et l'Église d'Écosse, au sein de l'association SOS (Sense Over Sectarianism), a notamment obtenu de la Millennium Commission, qui finance des projets avec l'argent de la National Lottery, une subvention d'un montant de 402000 livres sterling destinée à financer des projets individuels et locaux de lutte contre la haine religieuse. Une autre mesure récemment votée par le Parlement écossais s'inspire non pas des recommandations du groupe de travail sur la haine religieuse, mais d'une proposition de loi, la Protection from Sectarianism Bill, qui avait été déposée le 12 juin 2001 par un député libéral-démocrate, Donald Gorrie. La proposition visait à inclure dans la loi la notion selon laquelle le mobile religieux pouvait être retenu comme circonstance aggravante d'un crime ou d'un délit. Elle avait été abandonnée, mais l'idée sur laquelle elle reposait fut adoptée dès le 11 décembre 2002, soit quelques jours à peine après la publication du rapport sur la haine religieuse. Elle fut votée par l'une des deux commissions à la justice du Parlement écossais (la Justice 2 Committee), sous la forme d'un amendement à un projet de loi, le Criminal Justice (Scotland) Bill, qui était en cours d'examen à Édimbourg $^{16}$. Le jour du vote, Donald Gorrie avança plusieurs arguments en faveur de l'amendement. D'une part, la société écossaise semblait y être largement favorable. C'était le cas de l'exécutif, et notamment du Solicitor General for Scotland, l'un des deux juristes membres de l'exécutif (l'autre étant le Lord Advocate). C'était aussi le cas de l'ensemble des Églises écossaises, à l'exception de la Free Church of Scotland, qui est très minoritaire et qui, selon Gorrie, l'avait qualifié d' «antéchrist» pour avoir osé déposer une proposition de loi sur la haine religieuse. Gorrie affirma également avoir obtenu le soutien de la Law Society of Scotland, de l'association Nil By Mouth ou encore de plusieurs collectivités locales. D'autre part, l'un des principaux arguments avancés en faveur de l'adoption d'une loi ou d'un article de loi faisant du mobile religieux une circonstance aggravante était qu'une telle loi existait déjà dans le cas des crimes à caractère racial. L'amendement fut donc rédigé 
sur le modèle d'un article du Crime and Disorder Act concernant les crimes et délits à caractère racial et voté par le Parlement britannique en $1998{ }^{17}$. Quatre des sept membres de la commission parlementaire à la justice votèrent en faveur de l'adoption de l'amendement ${ }^{18}$. C'est donc sous sa forme amendée que le Criminal Justice (Scotland) Bill fut voté à la Chambre le 20 février 2003, et qu'il reçut l'assentiment royal le 27 juin 2003.

Linclusion d'un article sur les crimes et délits à caractère religieux dans le projet de loi sur la justice pénale avait été accueillie avec scepticisme par une partie des légistes écossais, qui avaient souligné l'inutilité de l'article dans un contexte où la haine religieuse était déjà prise en compte dans le jugement des délits et des crimes. En outre, beaucoup d'entre eux avaient été d'avis que le vote de cet article ne donnerait pas de résultats immédiats et qu'il faudrait beaucoup de temps avant que la police et les plaignants ne s'en saisissent. Toutefois, leurs prévisions se sont avérées infondées. Le 29 février 2004, le Crown Office a rendu publiques ses premières statistiques officielles concernant le nombre d'incidents d'origine sectaire qui lui avaient été rapportés par la police. Entre juin 2003, date du vote du Criminal Justice (Scotland) Act, et février 2004, ce sont 262 incidents d'origine sectaire, soit en moyenne plus d'un incident par jour, que la police a porté à l'attention des procureurs. Malgré tout, les Écossais restent sceptiques quant aux effets concrets que peuvent avoir des mesures d'origine institutionnelle, et a fortiori gouvernementale, sur les mentalités. Les efforts déployés par la police dans la lutte contre le sectarisme religieux, notamment pendant les matchs Rangers-Celtic, en contraignant par exemple les supporters du Celtic à attendre que tous ceux des Rangers aient évacué le stade avant de quitter les tribunes, ont bien contribué à réduire la violence physique pendant les matchs, mais non à réduire l'intolérance des spectateurs. De plus, il semblerait que les violences sectaires soient plus nombreuses à la sortie des pubs où les matchs sont retransmis qu'à la sortie des stades; c'est du moins ce qu'a affirmé Donald Gorrie en défense de l'amendement au Criminal Justice (Scotland) Bill. Or, si l'on peut sécuriser les stades et leurs abords, on ne peut faire surveiller chaque pub du pays. La police et la justice dénoncent également l'inapplicabilité de certaines des mesures gouvernementales. Labsence de statistiques officielles sur le sectarisme religieux jusqu'en juin 
dernier tenait par exemple à plusieurs facteurs. Du point de vue des autorités, il est beaucoup plus hasardeux de définir une agression à caractère religieux qu'une agression à caractère racial, par exemple, la haine religieuse étant difficile à isoler d'autres facteurs tels que la politique d'inspiration nord-irlandaise et l'identification nationale, à la République d'Irlande d'un côté, et au Royaume-Uni de l'autre. Peut-on considérer, par exemple, que chanter un hymne national ou faire le signe de croix constituent des agressions à caractère religieux? Imaginons un contexte dans lequel un supporter du Celtic attaque un homme portant un bonnet aux couleurs des Rangers et une écharpe arborant la Red Hand Of Ulster, qui orne le drapeau sectaire des protestants d'Irlande du Nord. Dans un tel contexte, qu'est-ce qui a motivé le supporter du Celtic à commettre cette agression: le fait que sa victime soutienne le mauvais club de football, qu'elle soutienne des positions politiques contraires aux siennes, ou qu'elle ne soit pas de même confession que lui? De plus, même si l'on juge que l'origine sectaire d'un meurtre ne fait aucun doute, notamment lorsqu'une personne est tuée pour avoir porté un maillot de football indiquant son appartenance religieuse, comment prouver le caractère sectaire d'une agression lorsqu'il n'y a pas de signe extérieur visible de cette appartenance religieuse? Toutefois, si l'on peut douter de l'efficacité à court terme des mesures préconisées par l'exécutif, il ne faut pas en minimiser l'importance symbolique. Les responsables politiques écossais ont trop longtemps ignoré le problème du sectarisme religieux, et c'est la mise en place d'un Parlement et d'un exécutif autonomes à Édimbourg en 1999 qui aura permis à la lutte contre le sectarisme de devenir l'un des grands chantiers nationaux écossais.

\section{ANNEXES}

\section{Annexe 1: Exemple de chant sectaire}

Hullo, Hullo

(extrait de la version originale)

Hullo, Hullo

We are the Rangers Boys

Hullo, Hullo

You'll know us from our noise

We'll give anything to see our team 
At Ibrox* or away

'Cause we are the Glasgow Rangers Boys

(*Nom du stade des Rangers.)

Hullo, Hullo (The Billy Boys)

(extrait de la version sectaire)

Hullo, Hullo

We are the Billy Boys

Hullo, Hullo

You'll know us from our noise

We're up to our knees in Fenian blood

Surrender or you'll die

'Cause we are the Glasgow Billy Boys

Annexe 2: $2^{\mathrm{e}}$ et $7^{\mathrm{e}}$ sections de l'article 74 du Criminal Justice (Scotland) Act de 2003 (voté au Parlement écossais)

2) An offence is aggravated by religious prejudice if:

a) at the time of committing the offence or immediately before or after doing so, the offender evinces towards the victim (if any) of the offence malice and ill-will based on the victim's membership (or presumed membership) of a religious group, or of a social or cultural group with a perceived religious affiliation; or

b) the offence is motivated (wholly or partly) by malice and illwill towards members of a religious group, or of a social or cultural group with a perceived religious affiliation, based on their membership of that group.

$[\ldots]$

7) In this section, «religious group» means a group of persons defined by reference to their-

a) religious belief or lack of religious belief,

b) membership of or adherence to a church or religious organisation;

c) support for the culture and traditions of a church or religious organisation; or

d) participation in activities associated with such a culture or such traditions. 
Annexe 3 : 2e section de l'article 33 du Crime and Disorder Act de 1998 (voté au Parlement britannique; article s'appliquant uniquement à l'Écosse) :

2) A course of conduct or an action is racially aggravated if-

a) immediately before, during or immediately after carrying out the course of conduct or action the offender evinces towards the person affected malice and ill-will based on that person's membership (or presumed membership) of a racial group; or

b) the course of conduct or action is motivated (wholly or partly) by malice and ill-will towards members of a racial group based on their membership of that group.

\section{Bibliographie succinte}

Bruce S., «Hooliganism Is the Real Source of the Problem» Herald, 6 décembre 2002.

Cross-Party Working Group on Religious Hatred, Tackling Religious Hatred, Édimbourg, The Stationery Office, 2002.

Devine T. M. éd., Scotland's Shame? Bigotry and Sectarianism in Modern Scotland, Édimbourg et Londres, Mainstream Publishing, 2000.

Mulholland M., Northern Ireland. A Very Short Introduction, Oxford, Oxford University Press, 2002.

Murray B., Bears, Bhoys and Bigotry. The Old Firm in the New Age, Édimbourg et Londres, Mainstream Publishing, 1998.

-, The Old Firm: Sectarianism, Sport and Society in Scotland, Édimbourg, John Donald Publishers, 2000.

Sanders, A., «Football and Sectarianism: A Background Picture», <http://www.realmaroonfc.com/documents/feat _sectarianism_l.htm $>$. 